\title{
Comparative Analysis of the Extracellular Matrix Composition in Proliferating and Involuted Infantile Hemangiomas
}

\author{
Hyochun Park ${ }^{1}$, Hannara Park ${ }^{1}$, Ho Yun Chung ${ }^{2}$, Teresa $\mathrm{M} \mathrm{O}^{3}$, Milton Waner ${ }^{3}$ \\ ${ }^{1}$ Department of Plastic and Reconstructive Surgery, Daegu Fatima Hospital, Daegu; ${ }^{2}$ Department of Plastic and Reconstructive Surgery, \\ Kyungpook National University School of Medicine, Daegu, Korea; ${ }^{3}$ Vascular Birthmark Institute of New York, Lenox Hill and Manhattan \\ Eye, Ear, and Throat Hospitals, New York, NY, USA
}

Background Changes in the composition of the extracellular matrix (ECM) occur between the proliferating and involuted phases of infantile hemangiomas $(\mathrm{IH})$, and are associated with angiogenic growth. We examined the composition of the ECM in proliferating and involuted $\mathrm{IHs}$ and assessed correlations between the composition of the ECM and whether the $\mathrm{IH}$ was in the proliferating or the involuted phase.

Methods We evaluated IH samples from a cohort of patients who had five proliferating IHs and five involuted IHs. The following ECM molecules were analyzed using enzyme-linked immunosorbent assays and immunohistochemistry: laminin, fibronectin, collagen type I, collagen type II, and collagen type III.

Results The involuted IHs had higher levels of deposition of collagen type III than the proliferating $\mathrm{IHs}$. The median values (interquartile ranges) were 1.135 (0.946-1.486) and 1.008 (0.7801.166) $(P=0.019)$, respectively. The level of laminin was higher in involuted IHs than in proliferating IHs, with median values (interquartile ranges) of 3.191 (2.945-3.191) and 2.479 (1.6993.284) $(P=0.047)$, respectively. Abundant collagen type III staining was found in involuted IHs. Laminin $\alpha 4$ chain staining was clearly present within the basement membrane adjacent to the blood vessels, and was significantly more intense in involuted IHs than in proliferative IHs. Conclusions Involuted hemangiomas showed extensive deposition of collagen III and laminin, suggesting that differences in the composition of the ECM reflect stages of the development of IHs. This pattern may be due to the rapid senescence of IHs.

Keywords Hemangioma / Extracellular matrix / Laminin / Collagen / Aging
Correspondence: Ho Yun Chung Department of Plastic and Reconstructive Surgery, Kyungpook National University School of Medicine, 130 Dongdeok-ro, Jung-gu, Daegu 41944, Korea Tel: +82-53-420-5692 Fax: +82-53-425-3879 E-mail: hy-chung@knu.ac.kr

Received: 16 Apr 2015 • Revised: 11 Jul $2015 \bullet$ Accepted: 23 Jul 2015

pISSN: 2234-6163 • elSSN: 2234-6171 • http://dx.doi.org/10.5999/aps.2015.42.5.544 • Arch Plast Surg 2015;42:544-551

This research was supported by the Basic Science Research Program through the National Research Foundation of Korea, funded by the Ministry of Education (2014R1A1A4A01009584).

This article was presented at the 5 th R\&R Forum on May 14, 2015 in Pyeongchang, Korea.

No potential conflict of interest relevant to this article was reported.

\section{INTRODUCTION}

Infantile hemangioma $(\mathrm{IH})$, which is a benign neoplasm of vascular endothelial cells, is the most common tumor that affects children [1]. Moreover, it is the most common lesion clinically diagnosed among newborns, with an occurrence rate of $4 \%-10 \%$
[2]. Hemangiomas are characterized by distinct phases of proliferation and regression, involving changes in the balance between proliferation and apoptosis in vascular endothelial cells. In the first phase, known as the proliferating period, the tumor progresses rapidly due to excessive endothelial cell proliferation over the course of six to ten months. The involuting phase fol- 
lows, which lasts for seven years. During this phase, endothelial cell hyperplasia is reduced and apoptosis increases, leading to spontaneous regression. At approximately the age of 10 years, the tumor enters its final phase, known as the involuted phase, which eventually involves complete regression, which is marked by the replacement of blood vessels with fibrofatty tissue [3]. Most hemangiomas remain relatively small and do not pose significant clinical problems. However, in a small number of patients, the tumors may grow rapidly to a size that can be problematic and even life-threatening. Moreover, $40 \%-80 \%$ of IHs leave a permanent scar or a mass of fat after the involution of the tumor, which can be pose significant aesthetic problems for children with facial lesions [4].

The extracellular matrix (ECM) plays a fundamental role in supporting the vascular endothelium. Primarily through adhesive interactions with endothelial cell integrins, ECM components provide the scaffolding that allows vascular endothelial cells to be organized into blood vessels. The diversity of ECM components in the endothelial cell microenvironment and of the mechanisms that regulate the synthesis and degradation of ECM components suggests that the ECM is sufficiently complex to exert significant and precise control over many aspects of neovascularization and blood vessel maturation [5].

The interactions between the ECM and cells, mediated by integrins on the endothelial surface, provide crucial environmental signals to endothelial cells that potentially control angiogenesis. The induction of vascular permeability through the loss of cellular adhesion, cell migration, and the secretion of proteolytic enzymes provides plasma-borne matrix molecules with access to extracellular spaces. Thus, the composition of the ECM may vary between sites of vascular formation and active angiogenesis. In one study, changes of the ECM environment were observed between the phases of $\mathrm{IH}$ and were associated with angiogenic growth, suggesting a causal link between angiogenesis and changes in the ECM composition [6]. Although all cases in this report showed a significantly higher level of some elements involved in the regulation of angiogenesis, those observations were not directly linked with a change of phase. The observation of changes in the ECM composition raises the question of its role in the transition between the proliferating and involuted stages, as well as the question of which characteristics of the ECM composition might predict the phase of an $\mathrm{IH}$. We therefore examined the composition of the ECM in the proliferating and involuted phases of IHs. The aim of this study was to evaluate differences in the ECM environment between proliferating and involuted IHs, with the ultimate goal of discovering how the composition of the ECM is associated with the transition between the phases of IHs.

\section{METHODS}

\section{Tissue sampling}

Hemangioma tissues were obtained via surgical excision in 10 patients, reflecting the proliferative phase of $\mathrm{IH}(\mathrm{n}=5$, patients aged 3-11 months) and the involuted phase of $\mathrm{IH}(\mathrm{n}=5$, patients aged 5.5-8.5 years). None of the children had received pharmacological therapy. All patients provided informed consent, and the study was conducted in accordance with the ethical guidelines of the Helsinki Declaration and received approval from the Institutional Review Board (KNU 2015-0055)". Chart review was combined with histological findings to determine whether the hemangiomas were in the proliferative or involuted phase.

\section{Enzyme-linked immunosorbent assay}

Enzyme-linked immunosorbent assay (ELISA) kits capable of determining collagen type I, II, III, fibronectin, and laminin levels were used on the proliferative and involuted IH tissue samples. ELISA was performed using kits for human collagen type I (Chondrex Inc., Redmond, Washington, USA), human collagen types II and III, laminin (USCN Life Science Inc., Wuhan, Hubei, China) and human fibronectin (Millipore Corp. Inc., Darmstadt, Hesse, Germany) in accordance with the manufacturer's instructions, $1 \mu \mathrm{g}$ of protein in $100 \mu \mathrm{L}$ of diluent was used to normalize the protein content across all samples. All samples were assayed in duplicate and the absorbance was then measured using an ELISA analyzer at $450 \mathrm{~nm}$. Statistical analysis was performed using the Mann-Whitney $U$ test, with the significance level set at 0.05 .

\section{Immunohistochemistry}

All tissues obtained from the 10 patients were $10 \%$ phosphatebuffered, formalin-fixed, and paraffin-embedded. The tissues were processed, cut at a thickness of $4 \mu \mathrm{m}$, stained with hematoxylin-eosin and Masson's trichrome, and evaluated by light microscopy. In addition, immunohistochemical staining with collagen type III, laminin $\alpha 4$, and laminin $\alpha 5$ antibodies was performed to evaluate expression patterns in the ECM composition of all hemangioma tissue samples. Tissue sections were cut at a thickness of $4 \mu \mathrm{m}$ and placed on Probe on Plus microscope slides (Fisher Scientific International Inc., Hampton, New Hampshire, USA). Using the Benchmark XT automated immunohistochemistry stainer (Ventana Medical Systems, Inc., Tucson, AZ, USA), slides were stained using the following procedure. The Ventana Ultraview DAB Kit (Ventana Medical Systems Inc., Tucson, Arizona, USA) was used for detection. Sections were deparaffinized using the EZ Prep solution contained 
in this kit. CC1 standard ( $\mathrm{pH} 8.4$ buffer containing Tris, borate, and/EDTA) was used for antigen retrieval for 60 minutes at $100^{\circ} \mathrm{C}$. A DAB inhibitor $\left(3 \% \mathrm{H}_{2} \mathrm{O}_{2}\right.$, endogenous peroxidase $)$ was used for blocking for four minutes at $37^{\circ} \mathrm{C}$. The slides were incubated with antibodies for collagen type III (Abcam Plc., Cambridge, Cambridgeshire, UK, diluted 1:100), laminin a4, and laminin $\alpha 5$ (Antibodies-online, Inc., Atlanta, Georgia, USA, diluted 1:100) for 32 minutes at $37^{\circ} \mathrm{C}$, and a secondary antibody of universal horseradish peroxidase multimer for eight minutes at $37^{\circ} \mathrm{C}$, followed by a $\mathrm{DAB}+\mathrm{H}_{2} \mathrm{O}_{2}$ substrate for eight minutes and a hematoxylin and bluing reagent counterstain at $37^{\circ} \mathrm{C}$. The reaction buffer ( $\mathrm{pH}$ 7.6 Tris buffer) was used as a washing solution. After staining, the slides were mounted and evaluated with a Leica DMI3000 light microscope (Leica Microsystems, Wetzlar, Germany). In each case, a representative area was digitally captured, with an image size of $3,888 \times 2,916$ pixels, using a Leica DFC420 digital camera and saved in the JPG format. The resulting images were analyzed using an I-solution image analyzer (IMT i-solution Inc., Vancouver, BC, Canada). Staining was quantified via measurement of the percentage area of red pixels, reflecting one of the three monochromatic pixel values.

\section{RESULTS}

\section{Enzyme-linked immunosorbent assay}

Collagen type I, collagen type II, and fibronectin levels were slightly higher in the involuted IH samples than in the proliferating IH samples. However, these differences were not statistically significant. The median value (interquartile range) for the collagen type III levels was 1.135 (0.946-1.486) in the involuted IH samples, compared to $1.008(0.780-1.166)$ in the proliferating $\mathrm{IH}$ samples. This difference was statistically significant $(\mathrm{P}=0.019)$. The median value (interquartile range) for the laminin levels was 3.191 (2.945-3.191) in the involuted IH samples, compared to 2.479 (1.699-3.284) in the proliferating IH samples. This difference was statistically significant $(\mathrm{P}=0.047)$ (Fig. 1).

\section{Histopathological findings}

Proliferating IHs are unencapsulated aggregates of closely packed, thin-walled capillaries, in which an endothelial lining is usually present. Blood-filled vessels are separated by minimal connective tissue. Histology of the proliferating IH samples showed a marked proliferation of capillaries in a lobular configuration (Fig. 2A, B). Histology of the involuted IH samples showed an absence of vascular proliferation as well as fibrofatty residuum, progressive fibrosis, and fat replacement of vascular tissue in the subcutis (Fig. 3A, B). The expression of collagen III and laminin $\alpha 4$ and $\alpha 5$ were analyzed by immunohistochemistry and an I- solution image analyzer. The expression of collagen III was significantly greater in the involuted phase than in the proliferative phase. Red pixels with values of 70 and 190 occupied $40.4 \%$ and $8.6 \%$ of the area of the slides, respectively (Figs. 2C, 3C). The expression of laminin $\alpha 4$ became diffuse and stronger along the basement membrane. The $\alpha 4$ chain staining was clearly present within the basement membrane adjacent to the vessels. The intensity of staining in the basement membrane was significantly greater in the involuted phase than in the proliferative phase (Figs. 2D, 3D). Red pixels ranging with values of 65 and 168 occupied $14.2 \%$ and $4.8 \%$ of the area of the slides, respectively. However, the expression of laminin $a 5$ was low in both phases, and no significant difference was observed regarding the expression of laminin $\alpha 5$ between the involuted and proliferating phases (Figs. 2E, 3E). Red pixels with values of 63 and 171 occupied $7.7 \%$ and $7.5 \%$ of the area of the slides, respectively.

\section{DISCUSSION}

In this study, we examined the composition of the ECM at different stages during the life cycle of IHs. We discovered that involuted hemangiomas showed augmented production of collagen III and laminin, as well as extensive deposition of $a 4$ laminin within the basement membrane adjacent to the vessels. The mechanisms that initiate the involution of IHs remain largely unknown. The increased apoptosis of endothelial cells and downregulated anti-apoptotic genes coincide with the onset of involution [7]. Aging progresses through a pathway involving apoptosis. Although it is still not clear whether the ECM regulates the apoptosis of cells in IHs, the different ECM compositions observed in our study suggest that laminin and collagen are related to the progression of IHs.

The collagens are the predominant proteins of the ECM [8]. Of the 28 different types of collagen, type I is the most abundant in the human body. Type III is the second most common collagen in the body and is found in association with collagen type I. Collagen type III has a similar pattern of distribution to collagen type I, but the ratio of these two collagens varies from tissue to tissue [9]. A relatively high ratio of collagen type III to type I is found in the cardiovascular system [10]. In addition, the quantity of collagen as a component of the ECM changes with aging. During skin development, the quantity of collagen type III in the ECM of the skin gradually increases [9]. The ratio of collagen type III to type I is related to aging and increases progressively as a component of various biosynthetic processes [11]. Our findings indicate that increased levels of collagen type III are part of how the composition of the ECM changes during the progression of IHs. Moreover, our data showing an increased 


\section{Fig. 1. Quantitative comparisons of the composition of the ECM using ELISA}

Levels of collagen type III and laminin were significantly higher in involuted IHs than in proliferating $\mathrm{IHs}$, which was a statistically significant difference; $P=0.019$ and $P=0.047$, respectively. $E C M$, extracellular matrix; $I H$, infantile hemangiomas. ${ }^{*} \mathrm{P}<0.05$.
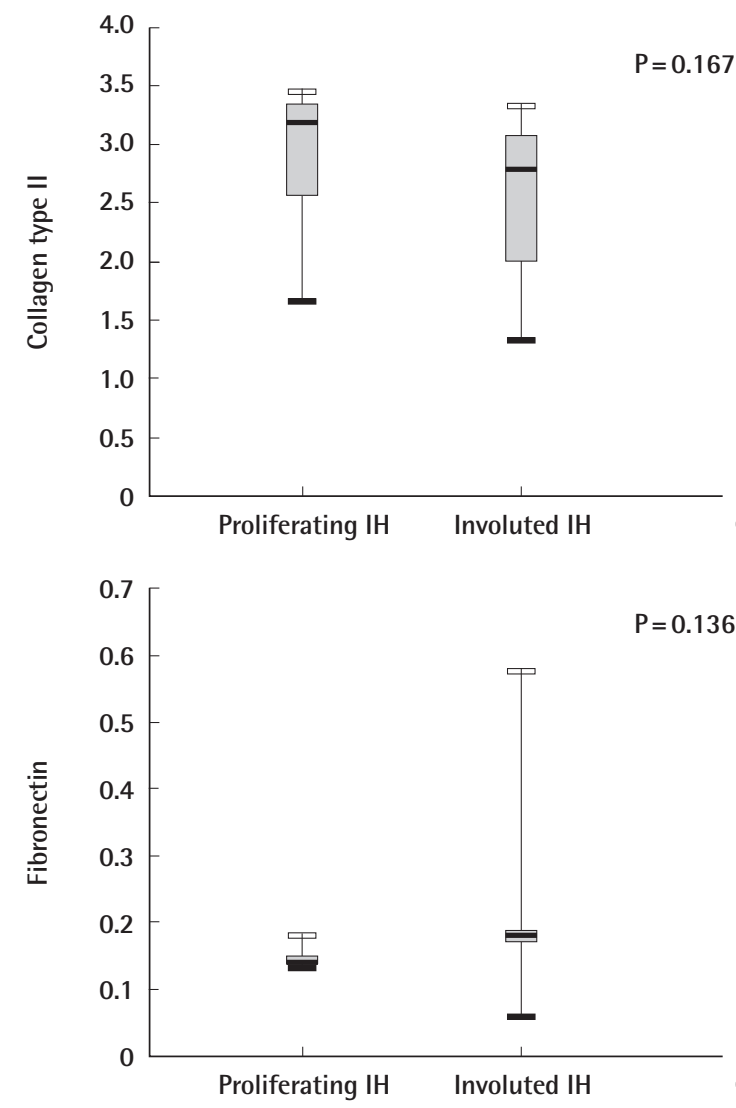

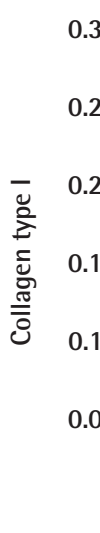

0.30
0.25
0.20
0.15
0.10
0.05
0
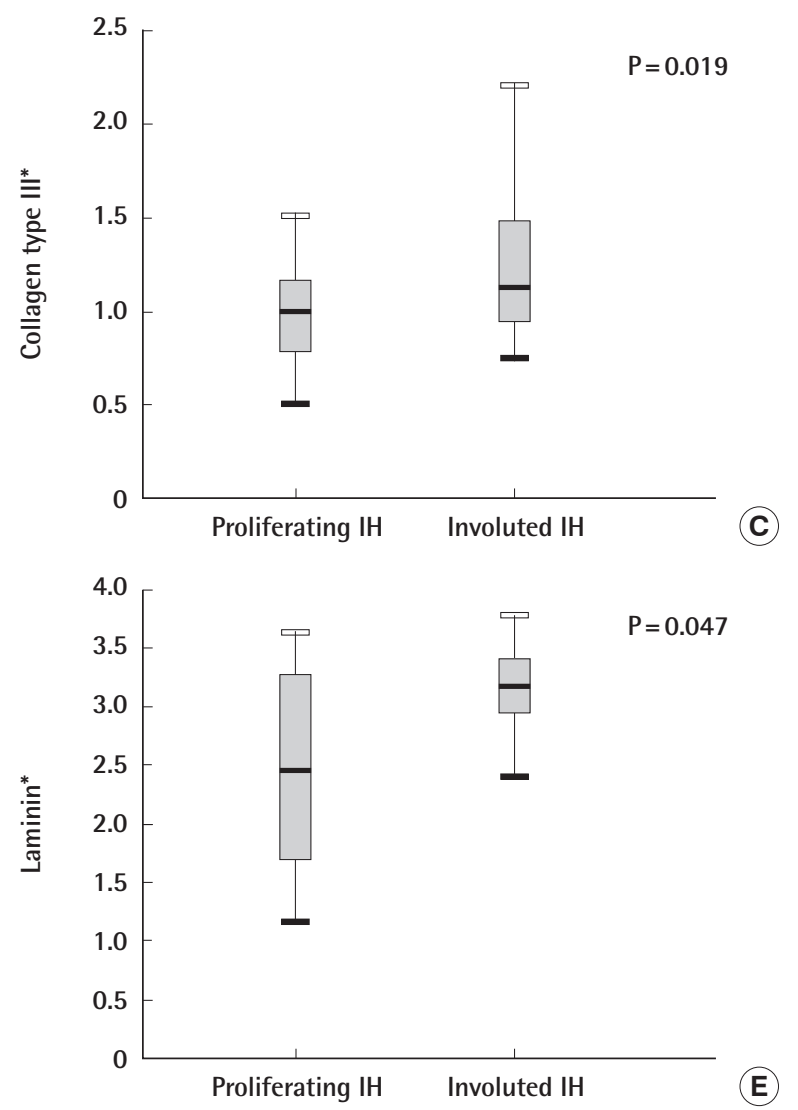

$P=0.133$

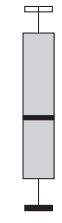

(A) deposition of collagen type III in involuted hemangiomas suggest that collagen type III is linked to aging.

Fibronectin is a large protein and an abundant component of the ECM. The expression of fibronectin is up-regulated during angiogenesis [12]. During the first four decades of human life, plasma fibronectin levels continuously increase, which may be associated with the synthesis of many ECM proteins in the liver. In individuals 40-82 years old, quantitative fibrin-, heparin-, or collagen-binding domain epitope reactivity was found to rise continually, whereas the expression of the cell-binding and carboxyl-terminal fibronectin domain was found to be at nearly the same levels as in younger subjects. Senescence and the progressive loss of bodily functions might be associated not only with age-dependent changes in plasma fibronectin molecular form but with the quantity of fibronectin [13]. In our study, we found no significant differences in fibronectin concentration between proliferating and involuted hemangiomas, suggesting that this parameter is independent of the phases of $\mathrm{IH}$.

Laminins are composed of three different types of chains, $\alpha, \beta$, and $\gamma$, which are usually found in the basement membrane. The trimeric combination of five $\alpha$, four $\beta$, and three $\gamma$ laminin chains results in a total of 15 different laminin isoforms. Laminins have 
Fig. 2. Histopathological findings characteristic of the proliferating phase

(A) The proliferating phase is highly cellular with immature vessels ( $H \& E_{\text {, }}$ $\times 200$ ). (B) Relatively scant connective tissue is present in the proliferating phase (trichrome stain, $\times 200$ ). (C) Anti-collagen III antibody was weakly stained (immunohistochemical stain, $\times 200$ ). (D) Laminin $\alpha 4$ staining was not observed (immunohistochemical stain, $\times 200$ ). (E) Laminin $\alpha 5$ antibody immunoreactivity was weakly present (immunohistochemical stain, $\times 200$ ).
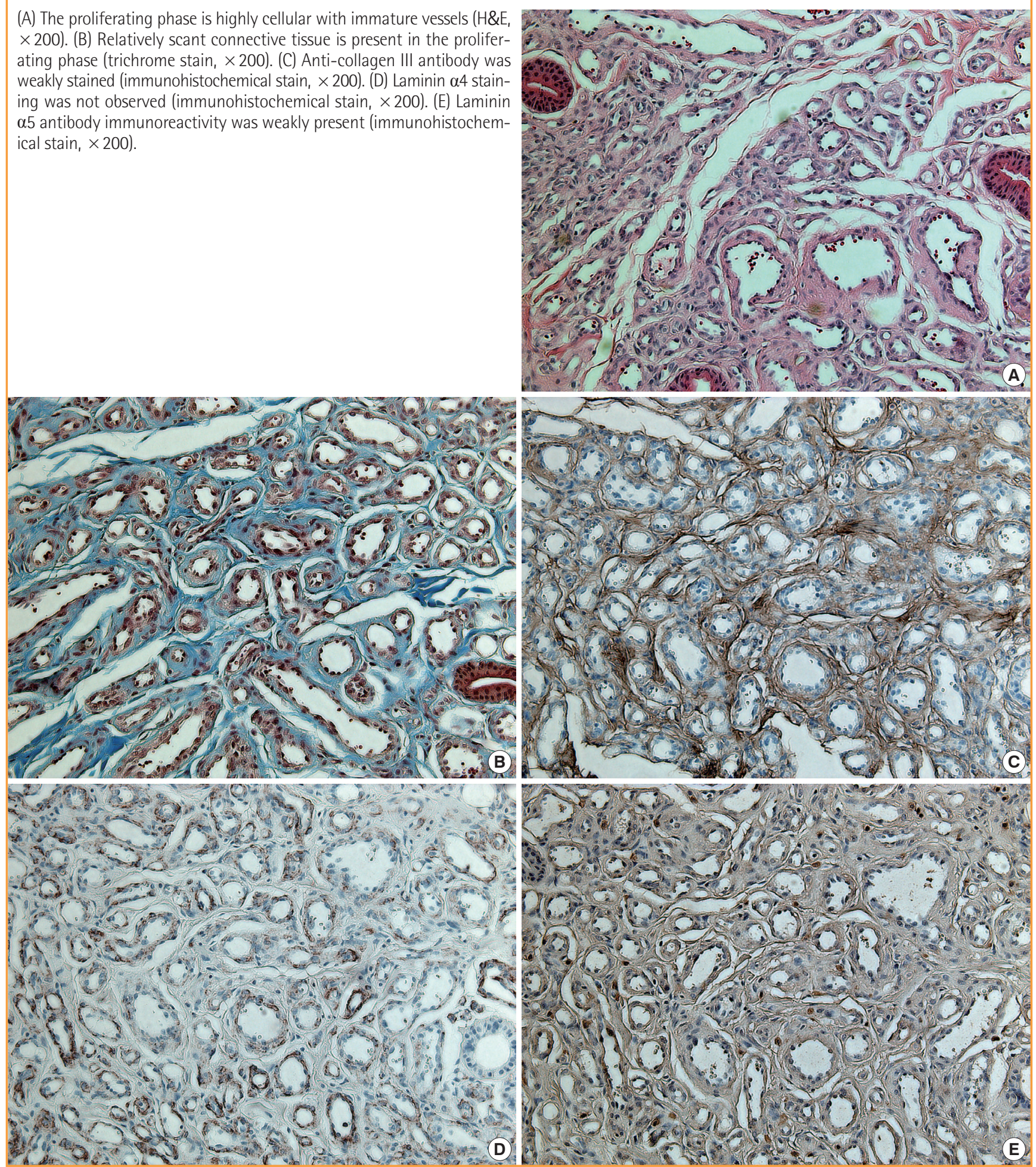

various sites for binding to receptors on the cell surface and to other ECM components, such as proteoglycans and collagens $[8,14]$. Vascular endothelial cells express two laminin chains, depending on the endothelial type and the phase of development (e.g., growth or activation). These two isoforms, laminin $\alpha 4$ and laminin $\alpha 5$, are the major laminins found in vascular basement membranes. These isoforms can combine with laminin $\beta 1$ and $\gamma 1$ chains in endothelial cell basement membranes to form laminin 8 and laminin 10, respectively. Laminin $\alpha 4$ is expressed by all vascular endothelial cells and is deposited in all 
Fig. 3. Histopathological findings characteristic of the involuted phase

(A) The involuted phase contains adipofibrous deposits and few remaining vessels $\left(H \& E_{1} \times 200\right)$. (B) Higher levels of deposition of connective tissue, including collagen, are present (trichrome stain, $\times 200$ ). (C) Tissues immunostained with anti-collagen III antibody, demonstrating extensive staining (immunohistochemical stain, $\times 200$ ). (D) The expression of anti-laminin $\alpha 4$ antibody is increased, especially in the basement membrane (immunohistochemical stain, $\times 200$ ). (E) The immunoreactivity of laminin $\alpha 5$ antibody was similar to that observed in the proliferating phase (immunohistochemical stain, $\times 200$ ).
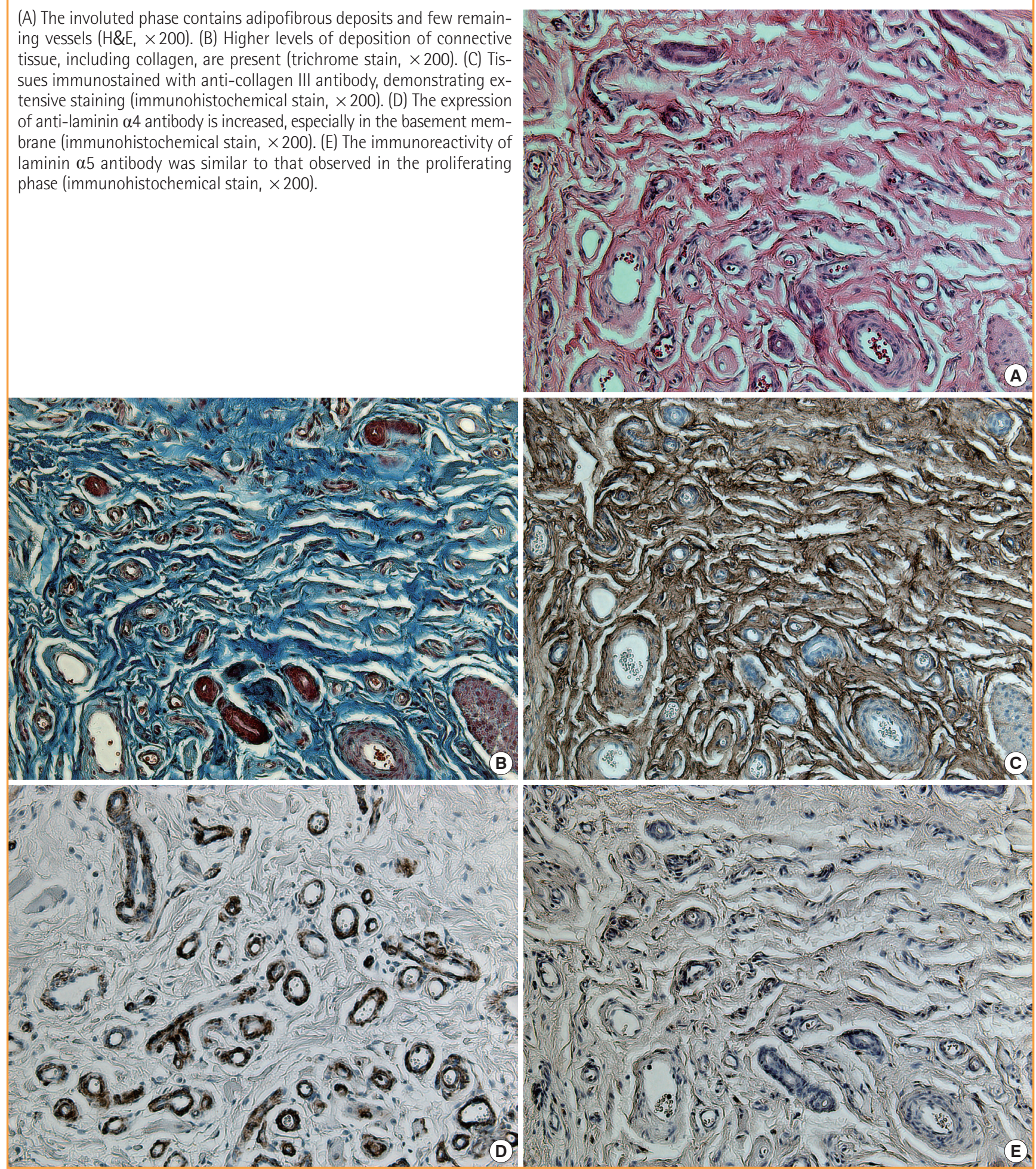

subendothelial basement membranes during all phases of vessel development. In contrast, laminin $\alpha 5$ is found primarily in the endothelial cell basement membranes of mature blood vessels, mostly in capillaries $[14,15]$. Increased laminin deposition may be related to the phase of vessel development. In our study, in- voluted hemangiomas showed a higher quantity of laminin $a 4$ in the basement membrane. Our results are consistent with the hypothesis that the involution of IHs might be related to the upregulation of laminin $a 4$ and, consequently, that involuted IHs may demonstrate extensive deposition of laminins. However, in 
our study, the expression of laminin $a 5$ was low in both phases, indicating that laminin $\alpha 5$ is not related to $\mathrm{IH}$ pathogenesis.

Vascular aging is associated with changes of both vascular function and structure that can take place at the level of vascular endothelial cells, smooth muscle cells, and the ECM of blood vessels [16]. One of the most relevant age-related vascular changes is a progressive intimal thickening composed of elongated cells and ECM accumulation with elastic tissue and collagen [17]. Similar to what is observed in large blood vessels, the age-related alteration of microvascular tone causes a progressive myogenic hypertrophic remodeling of small vessels, due to the altered distending pressure acting perpendicularly on the vessel wall $[18,19]$. Age-related changes in pericytes, the mural cells on capillaries, might also accelerate morphological and physiological deformities of the microvasculature. Age-related microvascular changes are characterized by broadening of the vessel and thickening of the basement membrane [20]. It has been found that basement membrane wall thickening is more frequent in vascular aging. This is consistent with our result that involuted hemangiomas showed a higher prevalence of thickening of the basement membrane than proliferating hemangiomas.

Aging is related to a decrease in the regenerative ability of endothelial cells and endothelial senescence, which is characterized by an increase in the quantity of endothelial cell apoptosis [21]. Although the increased apoptosis of endothelial cells and downregulated anti-apoptotic genes have some distinct features related to $\mathrm{IH}$ regression [7], the mechanisms that regulate apoptosis in IHs remain poorly understood. Apoptosis is strictly controlled by a complex interplay between B-cell lymphoma 2 proteins, caspases, and procaspases. During the proliferating phase of IH, the apoptosis rate is low. However, in the involuted phase, the rate of apoptosis increases by a factor of five, while levels of the apoptosis-inhibiting protein B-cell lymphoma 2 decrease [22].

Many questions about the transition between the phases of $\mathrm{IH}$ remain unresolved. The hemangioma life cycle is associated with several changes in the composition of the ECM and the morphology of the basement membrane. Involuted hemangiomas were found to show extensive deposition of collagen III and laminin, as well as basement membrane wall thickening adjacent to blood vessels, although not much is known regarding the causes of these changes. Changes in the composition of the ECM are associated with the aging of IHs. These observations suggest that the ECM composition changes due to the rapid senescence of IHs. Further research is required to understand precisely how the ECM regulates the apoptosis and senescence of cells in IHs. This study provides some of the first data regarding differences in the ECM associated with senescence in IHs.

\section{REFERENCES}

1. Kleiman A, Keats EC, Chan NG, et al. Evolution of hemangioma endothelium. Exp Mol Pathol 2012;93:264-72.

2. Szychta P, Stewart K, Anderson W. Treatment of infantile hemangiomas with propranolol: clinical guidelines. Plast Reconstr Surg 2014;133:852-62.

3. Boye E, Jinnin M, Olsen BR. Infantile hemangioma: challenges, new insights, and therapeutic promise. J Craniofac Surg 2009;20 Suppl 1:678-84.

4. Boscolo E, Bischoff J. Vasculogenesis in infantile hemangioma. Angiogenesis 2009; 12:197-207.

5. Davis GE, Senger DR. Endothelial extracellular matrix: biosynthesis, remodeling, and functions during vascular morphogenesis and neovessel stabilization. Circ Res 2005;97: 1093-107.

6. Jang YC, Arumugam S, Ferguson M, et al. Changes in matrix composition during the growth and regression of human hemangiomas. J Surg Res 1998;80:9-15.

7. Yu Y, Fuhr J, Boye E, et al. Mesenchymal stem cells and adipogenesis in hemangioma involution. Stem Cells 2006;24: 1605-12.

8. Rhodes JM, Simons M. The extracellular matrix and blood vessel formation: not just a scaffold. J Cell Mol Med 2007; 11:176-205.

9. Mohammadzadeh E, Nikravesh MR, Jalali M, et al. Immunohistochemical study of type III collagen expression during pre and post-natal rat skin morphogenesis. Iran J Basic Med Sci 2014;17:196-200.

10. Jensen LT, Host NB. Collagen: scaffold for repair or execution. Cardiovasc Res 1997;33:535-9.

11. Labat-Robert J, Kern P, Robert L. Biomarkers of connective tissue aging: biosynthesis of fibronectin, collagen type III, and elastase. Ann N Y Acad Sci 1992;673:16-22.

12. Antia M, Baneyx G, Kubow KE, et al. Fibronectin in aging extracellular matrix fibrils is progressively unfolded by cells and elicits an enhanced rigidity response. Faraday Discuss 2008;139:229-49.

13. Lemanska-Perek A, Pupek M, Polanska B, et al. Alterations in molecular status of plasma fibronectin associated with aging of normal human individuals. Clin Biochem 2013;46: 787-94.

14. Hallmann R, Horn N, Selg M, et al. Expression and function of laminins in the embryonic and mature vasculature. Physiol Rev 2005;85:979-1000.

15. Eble JA, Niland $S$. The extracellular matrix of blood vessels. Curr Pharm Des 2009;15:1385-400.

16. Matz RL, Schott C, Stoclet JC, et al. Age-related endothelial 
dysfunction with respect to nitric oxide, endothelium-derived hyperpolarizing factor and cyclooxygenase products. Physiol Res 2000;49:11-8.

17. Ferlosio A, Arcuri G, Doldo E, et al. Age-related increase of stem marker expression influences vascular smooth muscle cell properties. Atherosclerosis 2012;224:51-7.

18. Scioli MG, Bielli A, Arcuri G, et al. Ageing and microvasculature. Vasc Cell 2014;6:19.

19. Feihl F, Liaudet L, Levy BI, et al. Hypertension and micro- vascular remodelling. Cardiovasc Res 2008;78:274-85.

20. Hughes S, Gardiner T, Hu P, et al. Altered pericyte-endothelial relations in the rat retina during aging: implications for vessel stability. Neurobiol Aging 2006;27:1838-47.

21. Brandes RP, Fleming I, Busse R. Endothelial aging. Cardiovasc Res 2005;66:286-94.

22. Storch $\mathrm{CH}$, Hoeger PH. Propranolol for infantile haemangiomas: insights into the molecular mechanisms of action. $\mathrm{Br}$ J Dermatol 2010;163:269-74. 